\title{
HACER PARA PENSAR: IDEAS, ESPACIOS Y HERRAMIENTAS
} MAKE TO THINK: IDEAS, SPACES AND TOOLS

\author{
Volumen 12, Número 1 \\ Enero-Abril \\ pp. 1-21
}

Este número se publicó el 28 de febrero de 2012

Claudia Urrea Giraldo

Eleonora Badilla Saxe

Ximena Miranda Garnier

Danny Barrantes Acuña

Revista indizada en REDALYC

Revista distribuida en las bases de datos:

CATÁLOGO DE LATINDEX, IRESIE, CLASE, DIALNET, DOAJ, E-REVIST@S,

Revista registrada en los directorios:

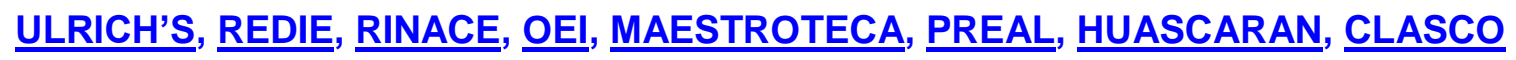




\title{
HACER PARA PENSAR: IDEAS, ESPACIOS Y HERRAMIENTAS MAKE TO THINK: IDEAS, SPACES AND TOOLS
}

\author{
Claudia Urrea Giraldo ${ }^{1}$ \\ Eleonora Badilla Saxe ${ }^{2}$ \\ Ximena Miranda Garnier ${ }^{3}$ \\ Danny Barrantes Acuña ${ }^{4}$
}

Resumen: Con el presente ensayo se busca compartir la experiencia de aprendizaje diseñada y desarrollada por un grupo interdisciplinario de profesores y profesoras de la Universidad de Costa Rica. La actividad contó con la participación de docentes de distintas disciplinas académicas. La propuesta se concretó en un taller que utilizó el lenguaje de programación Scratch y las tarjetas Pico, como oportunidad para modelar un ambiente de aprendizaje construccionista. Ideas poderosas, espacios y herramientas se pusieron a disposición de los y las participantes para la construcción de historias digitales, modelos y simulaciones. El objetivo final fue que los y las profesoras pudieran vivenciar, a través de una experiencia práctica, el ambiente de aprendizaje construccionista, las ideas y las herramientas, para que más adelante las pudieran extrapolar en sus propias disciplinas y cursos.

Palabras clave: CONSTRUCCIONISMO, LENGUAJE DE PROGRAMACIÓN SCRATCH, PICO BOARDS, DOCENCIA UNIVERSITARIA

Abstract: With this essay we intend to share a learning experience designed for and developed with an interdisciplinary group of faculty at the University of Costa Rica. Scratch programming language and Pico Boards supported this opportunity to experience a constructionist environment, where powerful ideas, spaces and tools were available for participants to design and construct their own digital stories, models and simulations. The final goal was that participants would experience, through a practical experience, the constructionist learning environment and the tools available, so that they could, in the future, extrapolate the knowledge they constructed in their own disciplines and classes.

Keywords: CONSTRUCTIONISM, SCRATCH, PICO BOARDS, UNIVERSITY LEARNING

\footnotetext{
1 Doctora en Medios, Artes y Ciencias, por el Instituto Tecnológico de Massachusetts MIT, Investigadora en el Media Lab, MIT. Directora de Aprendizaje de la Organización OLPC (One Laptop Per Child). Dirección electrónica: calla@media.mit.edu

${ }^{2}$ Doctora en Educación con énfasis en Mediación Pedagógica por la Universidad La Salle. Máster en Educación con énfasis en Tecnologías Digitales por la Universidad de Hartford. Catedrática de la Escuela de Artes Dramáticas de la Universidad de Costa Rica. Directora del Centro de Evaluación Académica. Dirección electrónica: eleonora.badilla@ucr.ac.cr

${ }^{3}$ Máster en Biología por la Universidad de Costa Rica y Máster en Educación por la Universidad de Harvard. Asesora de la Unidad de carreras transdisciplinares, Vicerrectoría de Docencia de la Universidad de Costa Rica. Dirección electrónica: ximena.miranda@ucr.ac.cr

${ }^{4}$ Máster en Medios Digitales de la Universidad de Bremen, Alemania. Profesor del Departamento de Docencia Universitaria Universitaria de la Escuela de Formación Docente y de la Escuela de Artes Plásticas de la Universidad de Costa Rica. Dirección electrónica: danny.barrantesacuna@ucr.ac.cr
}

Artículo recibido: 16 de setiembre, 2011

Aprobado: 26 de enero, 2012 


\section{Introducción}

El taller: "Ideas, espacios y herramientas: Hacer para pensar", ha sido una iniciativa de la Red Institucional de Formación y Evaluación Docente RIFED y de la Cátedra U de Transdisciplinariedad Complejidad y Ecoformación de la Vicerrectoría de Docencia de la Universidad de Costa Rica.

La iniciativa estuvo dirigida al profesorado universitario de diversas áreas y disciplinas con el objetivo de modelar un ambiente de aprendizaje construccionista. Para esto, se utilizó el lenguaje de programación Scratch y las tarietas Pico para que los y las participantes pudieran diseñar, de manera individual y colaborativa, historias digitales, modelos y simulaciones.

Tal como se presentará posteriormente, el Construccionismo es la propuesta educativa de Seymour Papert para la utilización creativa de las nuevas Tecnologías de la Comunicación y la Información (TIC) y la colaboración en el aprendizaje. El Lenguaje Scratch y las tarjetas Pico son herramientas digitales diseñadas en el Laboratorio de Medios (Media $\underline{\text { Lab) }}$ del Instituto Tecnológico de Massachusetts (MIT) por el grupo Lifelong Kindergarten que dirige el Dr. Mitchel Resnick.

En relación con los modelos y las simulaciones en educación son creaciones que se hacen con el propósito de ilustrar fenómenos científicos, obtener una mejor comprensión de un fenómeno observado, o de explicarles a otras personas ciertas ideas o datos con respecto a una investigación (Colella et al., 2001). Para estos autores, hay diversos tipos de modelos:

- llustrativos: son los que presentan procesos o sistemas científicos. Podría ser un ejemplo de las órbitas planetarias del sistema solar; modelos sobre las cadenas de ADN que se pueden manipular para enseñar la replicación o la transcripción, o cuerpos humanos transparentes que muestran los órganos internos. Todos estos capturan un aspecto de un sistema o proceso científico, y nos ayudan a comprenderlo en nuevas formas.

- Analíticos: se basan en ecuaciones matemáticas y permiten la exploración de una variedad de escenarios. Por ejemplo, un profesor de economía podría discutir un modelo de oferta y demanda que se describe por medio de ecuaciones. $O$ una profesora de física podría querer hacer un modelo sobre cómo en una ecuación dada, la posición depende de la aceleración y el tiempo. 
- De simulación: en lugar de resolver ecuaciones, se describen los mecanismos subyacentes y se deja que estos corran a través del tiempo para ver qué sucede. Con estos modelos, es más fácil incorporar eventos aleatorios y probabilísticos, reflejando aspectos importantes del mundo que nos rodea. Estas características de los modelos de simulación nos permiten exploraciones que son difíciles de alcanzar por medio de modelos analíticos, e imposibles de alcanzar con modelos ilustrativos.

Asimismo, el taller: "Ideas, espacios y herramientas: Hacer para pensar" fue diseñado, planeado y facilitado por un grupo interdisciplinario de docentes universitarios, para que fuera aprovechado por un grupo también interdisciplinario de docentes universitarios. Se llevó a cabo del 16 al 20 de mayo de 2011 y participaron 16 profesores y profesoras provenientes de: Informática, Evaluación, Comunicación, Economía, Recursos Humanos, Administración Educativa, Derecho, Sociología, Arquitectura, Matemática, Química, Medicina, Geografía e Historia.

El propósito general del taller fue que los y las profesoras pudieran vivenciar, a través de una experiencia práctica, el ambiente de aprendizaje construccionista, las ideas y las herramientas, para que más adelante las pudieran extrapolar en sus propias disciplinas y cursos.

\section{Sobre Construccionismo: hacer para pensar}

Basándose en las ideas constructivistas de $\underline{\text { Jean Piaget y del pensamiento de Lev }}$ Vygotsky, el reconocido pensador Seymour Papert propuso el Construccionismo como una visión educativa innovadora sobre el uso de las tecnologías digitales para apoyar el aprendizaje de las personas. Pero más allá de esta visión, el Construccionismo permite comprender la forma en que la tecnología digital es apropiada por la sociedad y por el individuo (Papert, 1990). Para el autor, el conocimiento es algo que se construye en la mente, mientras se construye algo tangible en el mundo físico y que, además, debe ser significativo (Papert, 1990).

En esta visión educativa, Papert otorgó a los y las aprendices un rol activo creativo, colocándolos como diseñadores de sus propios proyectos y constructores de su propio aprendizaje. Se trata de facultar ("empower") a los y las aprendices para que asuman ese papel activo. En contraposición a la instrucción asistida por computadora (CAI por sus siglas 
en inglés), que promueve que la computadora enseñe y programe al usuario, Papert propuso que sea el aprendiz quien programe a la computadora, ya que al hacerlo adquiere: "un sentido de dominio sobre un elemento de la tecnología más moderna poderosa y a la vez establece un íntimo contacto con algunas de las ideas más profundas de la ciencia, la matemática y el arte de construcción de modelos intelectuales" (Papert, 1987, pp.17-18). Papert dice que el mejor aprendizaje no vendrá al encontrar las mejores maneras de instruir: "sino de proveer a los estudiantes con las mejores oportunidades para construir" (en Fabel, 1990, p. 2).

Estas premisas implican que las personas poseen una habilidad natural para aprender de la experiencia, creando estructuras mentales que les permiten organizar y combinar la información y el conocimiento que se va construyendo en el transcurso de la vida. De acuerdo con Papert, el conocimiento se construye de manera especialmente fructífera cuando el aprendiz se involucra de manera consciente en una construcción pública que puede ser exhibida, discutida, probada, examinada o admirada (Flabel, 1990, p. 2-3). En ese sentido, Papert advierte que para hacerlo no es suficiente pedirle al estudiante que se haga cargo de su propio aprendizaje: es necesario instrumentalizarle con las herramientas adecuadas para que lo haga.

Asimismo, Papert (1990) dice que el Construccionismo es más que aprender haciendo. Plantea que es hacer con una motivación intrínseca; hacer con el impulso de los propios valores y deseos; hacer con comprensión de lo que se hace. Sobre todo, dice, es apropiarse del conocimiento; es hacerlo propio. Dice que esto se logra cuando las herramientas de construcción se hacen invisibles y el aprendiz se concentra en su propio aprendizaje y conocimiento

\section{Sobre dos ideas poderosas: Fluidez Tecnológica y Colaboración}

Un ambiente de aprendizaje construccionista se enfoca en la exploración y la construcción de ideas poderosas, además de la reflexión y la articulación de las mismas (Papert, 2000). Las ideas poderosas no son importantes por estar ubicadas dentro un marco curricular, sino porque dan al aprendiz la autonomía para abordar un tema y profundizar en él a través de un proceso concreto de construcción que lo pone en contexto. Algunas herramientas construccionistas de aprendizaje permiten la exploración de ideas poderosas de gran alcance: pensamiento algorítmico (Logo: Papert, 1987), pensamiento 
descentralizado (StarLogo: Resnick, 1994), mentoría (MOOSE Crossing: Bruckman, 1998), valores morales (Zora: Bers, 2001), colaboración y re-mezcla (Maloney, Resnick at al, 2010; Seneviratne, y Monroy-Hernández, 2010).

En el taller en cuestión se tomó como reto proponer situaciones en las cuales los participantes podían experimentar algunas ideas poderosas, pero incluso la más poderosas de todas: la idea de ideas poderosas. El interés central en esta oportunidad se vio complementada con dos de ellas: fluidez tecnológica y colaboración. Ambos ejes no solo son parte importante del propio proceso de aprendizaje en que estaban inmersos los docentes/aprendices, sino que también nutren el diseño y desarrollo de las herramientas de aprendizaje construccionistas que se estaban utilizando: Scratch y Pico Crickets (que se explican adelante).

\section{Fluidez tecnológica}

La fluidez tecnológica tiene que ver con el uso y la apropiación de herramientas tecnológicas para hacer o construir una tarea; para crear, comunicar y diseñar. Tal como la describen Papert y Resnick (1995), la fluidez tecnológica significa mucho más que la capacidad de utilizar herramientas tecnológicas que serían el equivalente a entender algunas frases comunes en un idioma. Para ser verdaderamente fluido en un idioma (como el inglés o el francés), la persona debe ser capaz de articular una idea compleja o contar un historia "cautivadora", es decir, debe ser capaz de "hacer cosas" con el lenguaje.

Analógicamente, el concepto de fluidez tecnológica implica no sólo saber cómo utilizar las herramientas tecnológicas, sino también saber cómo construir cosas significativas con esas herramientas. Una persona tecnológicamente fluida debe ser capaz de ir desde el origen de una idea intuitiva, a la aplicación de un proyecto tecnológico. La fluidez tecnológica tiene que ver, además, con la habilidad de programar, lo cual extiende las posibilidades de lo que se puede crear, y de lo que se puede aprender. Permite reflexionar acerca del propio pensamiento, incluso a pensar acerca de la actividad misma de pensar. Para Resnick y el grupo de Scratch: "la fluidez tecnológica implica diseñar, crear y re-mezclar, no solo navegar, conversar e interactuar" (Resnick et al., 2009, p. 60). Por eso, Scratch fue creado como un ambiente de aprendizaje:

1. De piso bajo o de fácil acceso, lo que implica que sus usuarios pueden fácilmente usar esta herramienta de aprendizaje construccionista aunque no tengan 
experiencia previas en programación.

2. De techo alto, lo cual quiere decir que los proyectos pueden llegar a ser más complejos a medida que los usuarios adquieren experiencia y fluidez con esta herramienta.

3. De paredes anchas, que permite la creación de diferentes tipos de proyectos, lo cual involucra personas con diferentes intereses y formas de aprender.

\section{Colaboración}

El diseño de Scratch, como herramienta de aprendizaje, viene acompañado de su propio sitio web. El grupo del Media Lab del MIT, que diseñó esta herramienta, está convencido que: "para [su] éxito, el lenguaje debe estar vinculado a una comunidad donde la gente puede apoyar, colaborar, criticar y construir a partir del trabajo de otros" (Resnick et al., 2009). La colaboración, además de ser una idea poderosa que se promueve desde el diseño y la experiencia con Scratch, fue parte medular del taller.

La interfaz de la herramienta de aprendizaje Scratch también fue diseñada con el propósito de facilitar la colaboración. Por ejemplo, en la página se puede encontrar el botón de "compartir", lo que significa que toma un solo "click" para hacerlo. De igual modo, cuando una persona toma la decisión de compartir su proyecto en el sitio web de Scratch, este queda a disposición de los demás usuarios. Ellos pueden no solamente verlo e inspirarse en él: pueden usarlo, reutilizarlo, calificarlo y valorarlo. Esta retroalimentación de múltiples vías, va conformando una comunidad y se convierte en una de las más grandes motivaciones para crear y compartir proyectos. Los miembros de la comunidad están constantemente adaptando y creando proyectos, a partir de las ideas de otros. La reutilización y enriquecimiento de proyectos es conocido como "remezcla". La información de cuántas veces y quién ha remezclado un proyecto está disponible en el sitio.

Por su parte, Scratch ha sido traducido a más de 40 idiomas, lo que ha permitido la colaboración a nivel internacional. La infraestructura de Scratch posibilita no solo la traducción en varios idiomas, sino también el uso de cualquier tipo de caracter.

En el taller realizado en la Universidad de Costa Rica, las y los participantes trabajaron en grupos en el diseño y en la creación de sus proyectos de Scratch. Asimismo, pudieron compartir sus proyectos en el sitio web, lo que les permitió una visibilización internacional. Algunos de los sub grupos se inspiraron en proyectos disponibles en el sitio web de Scratch 
para conceptualizar y diseñar los suyos propios. En esta ocasión, y por lo limitado del tiempo, la comunicación y la retroalimentación entre los y las participantes no se dio a través del sitio, pero sí se realizó presencialmente. Cada uno de los sub grupos tuvo la oportunidad de compartir sus ideas, recibir retroalimentación de otros, y reflexionar a partir de ella para el mejoramiento de sus proyectos. Adicionalmente, todos pudieron "tomar prestadas" ideas de los demás para incorporar en sus diseños.

\section{Sobre las herramientas: el Lenguaje de Programación Scratch ${ }^{5}$ y las Pico Boards}

Tal como mencionamos antes, tanto el Lenguaje de Programación Scratch como las Pico Boards son herramientas digitales diseñadas en el Laboratorio de Medios (Media Lab) del Instituto Tecnológico de Massachusetts ( $\underline{\mathrm{MIT}})$ por el grupo Lifelong Kindergarten que dirige el Dr. Mitchel Resnick.

Se trata de herramientas que heredan las ideas construccionistas de Papert y derivan de propuestas anteriores como el Lenguaje $\operatorname{Logo}^{6}$ y los ladrillos programables ${ }^{7}$, también del Media Lab.

La idea de Resnick y su grupo (2009) es proveer no solamente herramientas informáticas poderosas para el aprendizaje de las nuevas generaciones, sino todo un ambiente de apoyo y colaboración mutua.

El Lenguaje de Programación Scratch toma ideas de lenguajes de programación profesionales como Flash/ActionScript, o de otros como Alice7 and Squeak Etoys5 desarrollados específicamente para programadores jóvenes. Pero sus diseñadores buscaron un umbral de entrada más accesible para el aprendizaje de la programación, con más diversidad de opciones para desarrollar el pensamiento lógico computacional. De acuerdo con Resnick (2009) diseñaron un lenguaje de computación que fuera más traveseable ${ }^{8}$, más significativo y más sociable que otros lenguajes de programación.

Por eso, la gramática del lenguaje Scratch está basada en un conjunto de bloques digitales programables que se ensamblan unos con otros, tal como lo hacen bloques físicos

\footnotetext{
Scratch se puede descargar gratuitamente de la página http://scratch.mit.edu Versiones comerciales del Lenguaje Logo se han Ilamado LogoWriter y Micromundos. Versiones comerciales conocidas como Mindstorms y PicoCrickets.

Tinkerable es la palabra que utiliza Resnick en inglés.
} 
con los que juegan niños, niñas y jóvenes. Los bloques programables de Scratch están diseñados para que se ensamblen solamente si juntos tienen un sentido sintáctico. Las estructuras de control del lenguaje ("como repetir" y "por siempre") tienen una forma de C que indican que los bloques programables deben colocarse dentro. Los bloques que producen valores tienen forma dependiendo del valor que regresan (óvalos para los números y hexágonos para las funciones booleanas). Los bloques de los condicionales ("como si”, "o repetir hasta") tienen forma hexagonal incompleta, indicando que una función booleana se requiere.

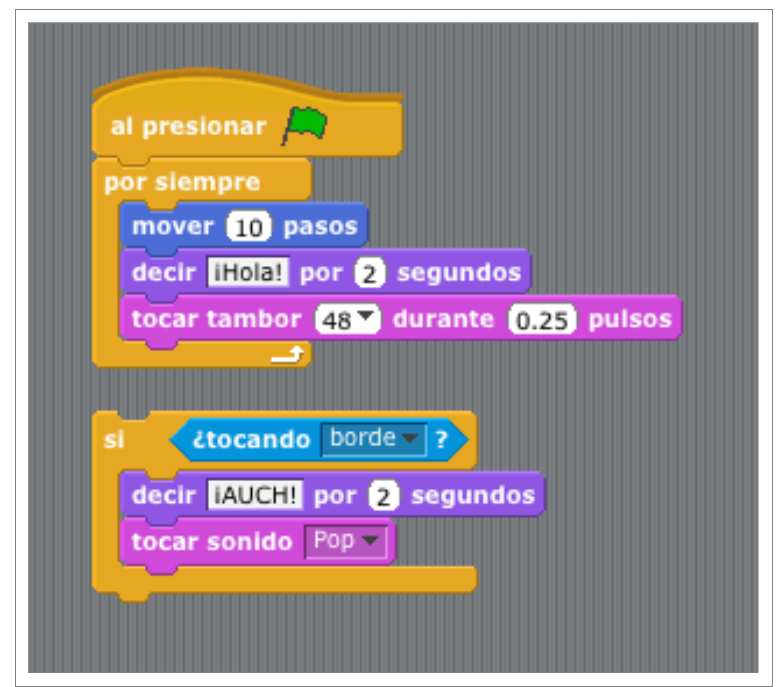

Figura No.1 Ejemplos de programación en Scratch.

Scratch además de ser un lenguaje de programación, también busca satisfacer la diversidad de estilos de aprendizaje, razón por la cual promueve que lo puedan utilizar tanto programadores que planean de manera vertical (de arriba hacia abajo), como quienes prefieren travesear con un pensamiento y planeamiento de abajo hacia arriba. De igual forma, la actividad de programación de este lenguaje consiste en mezclar gráficos, animaciones, fotos, música y sonidos. 


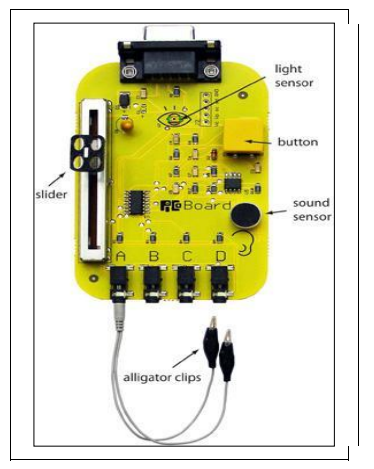

Figura No.2 Pico Board

Las Tarjetas Pico o Pico Boards (Figura No.2) son unas tarjetas electrónicas que permiten que los proyectos programados con Scratch interactúen con el mundo físico, por medio de sensores de luz y sonido. Tienen también un deslizador que controla el valor de las entradas (resistencia), un botón que reacciona al presionársele, un par de sensores que detectan sonido y luz. Además como parte del paquete disponible al público, se incluye un par de prensillas que permiten transmitir información externa variada al procesador interno del dispositivo. Estas tarjetas, por lo tanto, permiten que los proyectos en la pantalla reaccionen ante estímulos (de luz, de contacto, de resistencia o de sonido) que les llegan del mundo externo, permitiendo así diseñar proyectos interactivos.

\section{Sobre el diseño y desarrollo del taller}

El diseño de una actividad educativa construccionista requiere de una cuidadosa planificación previa para garantizar un marco de referencia muy sólidamente fundamentado y, a la vez, muy flexible. Esa combinación de solidez conceptual y flexibilidad metodológica es lo que añade complejidad al diseño construccionista de un ambiente de aprendizaje. Pero solamente con una cuidadosa planeación es que se lograr dar cabida a los emergentes y a la participación activa de todas las personas involucradas, sin que se corra el riesgo de promover un activismo sin sentido. En este contexto y en concordancia con las ideas de Papert: "objetos con los cuales pensar" deben estar a disposición, y la construcción de proyectos significativos por parte de los y las participantes es la estrategia fundamental de aprendizaje. De allí el nombre del taller (título de este artículo): hacer para pensar (Ver Anexo No.1). 
Con estas ideas en mente, se propuso una combinación dinámica de charlas sobre ideas poderosas (en cuanto al Construccionismo, así como sobre el potencial de lenguaje Scratch y las Pico Boards); demostraciones; ejercicios guiados; diseño y construcción de proyectos individuales y grupales, y sesiones de reflexión y reconstrucción de las experiencias y los aprendizajes (Figura No.3). Se optó por el título: "Ideas, herramientas y espacios", porque esto refleja el espíritu o visión pedagógica del taller: sería un espacio para el encuentro entre las ideas y las herramientas para hacerlas realidad.

El taller se desarrolló en un laboratorio de computación de la Escuela de Ciencias de la Computación e Informática (ECCI) de la Universidad de Costa Rica (UCR) durante cuatro días (lunes 16, martes 17, jueves 19 y viernes 20 de mayo del 2011). Se trabajó por cuatro horas cada día (de 1:00 a 5:00p.m.) separando los bloques de trabajo por un receso y refrigerio. Las fechas del taller fueron muy cercanas a la celebración del día mundial de Scratch (21 de mayo 2011), por lo cual la actividad se inscribió como parte de las actividades de Costa Rica en el día de Scratch.

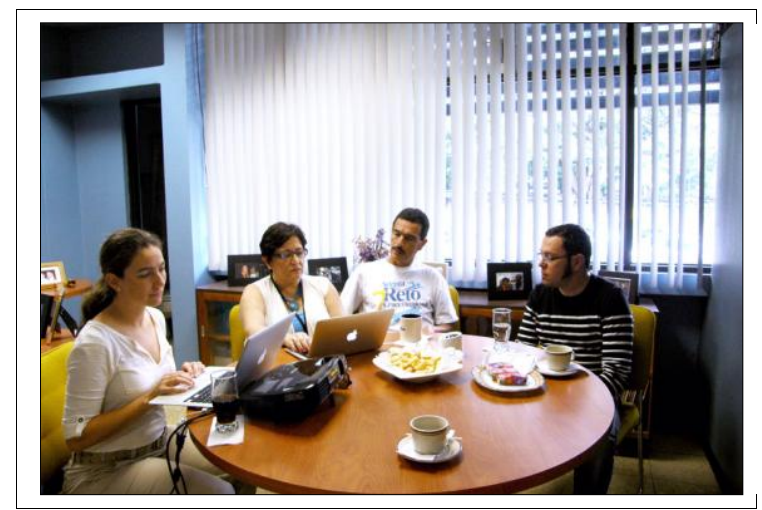

Figura No.3 Sesiones de trabajo, equipo organizador

El taller inició con una introducción titulada: “El espíritu del taller", donde se utilizó un proyecto hecho con Scratch para describir la intención de crear un espacio en el que todas las personas participantes (aprendices y mediadores) tuvieran la oportunidad y las herramientas para "hacer para pensar" en conjunto. Scratch, las tarjetas PICO y los materiales de construcción (incluyendo materiales de desecho) se presentaron como las herramientas que, en este caso, permitirían pensar y hacer juntos sobre formas de mejorar la 
docencia universitaria (Figura No.4). El construccionismo, la colaboración, la fluidez tecnológica, y la creación de modelos, simulaciones e historias digitales, se presentaron como las ideas que se colocaron en este espacio para incentivar la innovación en los espacios de aprendizaje universitarios. En otras palabras, en esta etapa se presentó brevemente la visión pedagógica del taller.

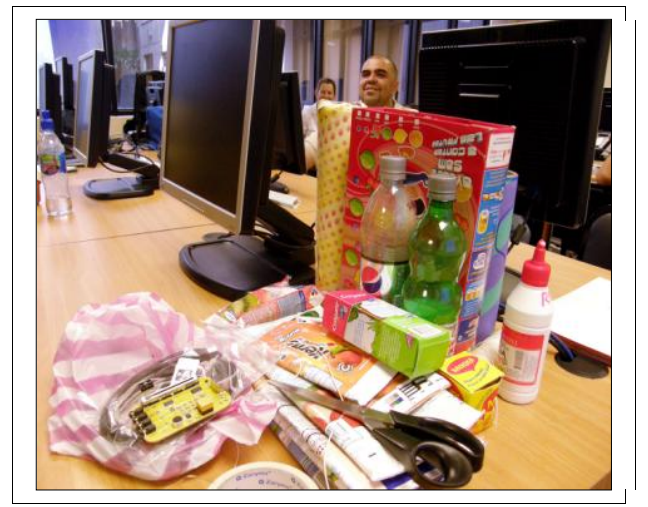

Figura No.4 Materiales de construcción y tarjetas PICO

A continuación, se hizo una primera demostración del funcionamiento básico de Scratch, seguida de una exploración libre de proyectos hechos con Scratch presentes en la galería original de este lenguaje de programación. El segundo y tercer día se iniciaron con sesiones demostrativas similares, en las cuales, además, se presentó el funcionamiento de las tarjetas PICO y cápsulas de conceptos de programación útiles. En el caso de la demostración de las tarjetas PICO, se utilizó el proyecto "sensorboard 1-sunrise" de la carpeta de ejemplos sobre motores y sensores que viene originalmente con Scratch, con una casita construida con cartulina y materiales de desecho (Figura No.5).

Después del espacio de exploración libre de Scratch, se realizó una dinámica en la cual se recogieron las primeras impresiones e ideas iniciales de los participantes. Dentro de la lógica programática del taller, este primer momento de encuentro resultó vital en la medida que permitió crear una atmósfera propicia y evocativa. Posibilitó, además, que los participantes y facilitadores encontrar líneas convergentes de interés y expectativas concretas para el desarrollo del taller. El ejercicio fue intencionado de manera que se pudiera apuntar hacia una construcción contextual de la realidad que compartía el conjunto de interlocutores. Como es mencionado por Resta: 
el comportamiento, el pensamiento y el contenido son las bases que estructuran la enseñanza y el aprendizaje -al menos en lo que respecta a la enseñanza organizada-. Estos elementos estructurales se vuelven accesibles al trabajar en el diseño de realidades «auténticas» en las prácticas de clase. (Resta, 2004, p.184)

El nodo problematizador que se propuso apuntó a la discusión de las distintas expectativas que se tenían del taller. La línea de desarrollo se hizo en función de un posible proyecto o tema y el impacto esperado en el devenir docente de cada participante. Se solicitó, asimismo, que escribieran sus respuestas en un trozo de cartulina que fue adherido a la pizarra junto con el resto de participaciones. Una vez reunidas las opiniones, se inició una discusión para reunir ideas comunes, principalmente las relacionadas con las tres áreas temáticas: historias digitales, simulaciones y modelos. Los participantes socializaron diversas impresiones que enriquecieron el debate y facilitaron la conformación de equipos de trabajo para el proceso que se desarrolló a lo largo de la semana (Figura No.6).
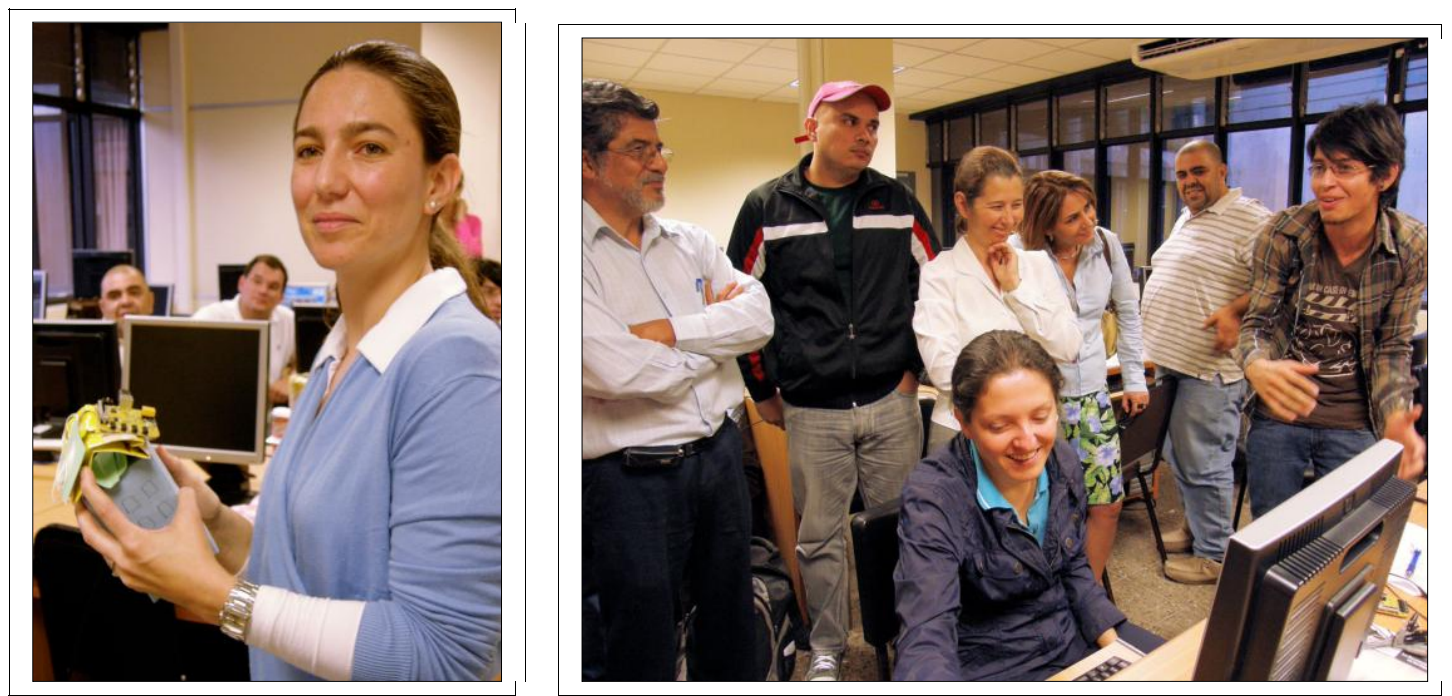

Figura No.5 Demostración de tarjeta PICO. Figura No.6 Discusión de trabajo en equipos

El primer y segundo día, se hicieron presentaciones sobre ideas poderosas seguidas de una plenaria abierta. Los temas desarrollados siguieron líneas temáticas que fueron abordadas y discutidas incluso desde el inicio del taller. Igualmente, se presentaron nuevos ejes de enriquecimiento teórico-práctico: la fluidez tecnológica y la colaboración, su importancia actual, y las formas que estas toman con Scratch. Tal como se ha señalado 
anteriormente, el espíritu e intencionalidad en el diseño de este espacio de formación fue la construcción de un escenario formativo que permitiera la inmersión en un ambiente construccionista.

Este ambiente sería innovador desde su naturaleza al desarrollar una configuración con elementos no tradicionales para un grupo interdisciplinario de docentes universitarios. La herramienta, Scratch, si bien es cierto representó un atractivo importante, fue siempre asumida y promovida como un vehículo cognitivo que permitió diferentes niveles de interacción. En el decir de Papert, se convirtió en un: "objeto con el cual pensar". 9

Tal como lo describe Cabero:

en definitiva, lo que queremos decir es que no serán los determinantes técnicos del sistema los que marcarán su calidad y su eficacia, sino la atención que les prestemos a las variables educativas y didácticas que se ponen en funcionamiento. Los problemas hoy no son tecnológicos, sino que se derivan de saber qué hacer y cómo hacerlo, y por qué queremos hacerlo. (2006, p. 8).

Al final del segundo día, se formaron cuatro grupos de trabajo, y se dio un espacio para que comenzaran a plantear un proyecto grupal con Scratch y las tarjetas Pico. Los días 3 y 4 se dedicaron principalmente al desarrollo de estos proyectos. Dos de los grupos se enfocaron en el desarrollo de proyectos de simulación, mientras que los otros dos crearon historias digitales.

Por el enfoque construccionista del taller, después de cada sesión de trabajo grupal se compartieron los aprendizajes en dinámicas en las que cada grupo mostraba a los demás sus ideas, avances y dificultades. Así, se permitió que se diera un mayor intercambio de ideas y aprendizajes entre los participantes, a partir de un proceso de reflexión sobre el trabajo realizado en los proyectos. De igual importancia fue cerrar cada uno de estos días con reflexiones y una reconstrucción del proceso vivido.

Al final del taller, se hizo una demostración sobre la forma de integrar a la comunidad internacional de Scratch por medio de la página web (www.scratch.media.mit.edu). Los proyectos de los participantes se compartieron con la comunidad internacional a través de este medio.

9 "Object to think with". 


\section{Sobre los hallazgos}

Debemos iniciar esta sección recordando que a pesar de que las herramientas tecnológicas jugaron un papel importante en este taller siempre lo hicieron como objetos con los cuales pensar. El fin fue experimentar y reflexionar sobre un ambiente de aprendizaje construccionista, como una alternativa pedagógica a la tradicional enseñanza universitaria.

\section{Las expectativas y primeras reacciones}

La mayoría de los y las participantes iniciaron el taller con la expectativa de buscar formas innovadoras de pedagogía, realistas y aplicables en los cursos; también de buscar nuevas formas de acercarse, y acercar sus estudiantes, al conocimiento, y de adquirir más instrumentos para hacer más atractiva sus clases, al permitir que sean los estudiantes quienes lideren los procesos de construcción de conocimiento. Las primeras reacciones se pidieron a los y las asistentes en forma de una frase corta. Algunas fueron:
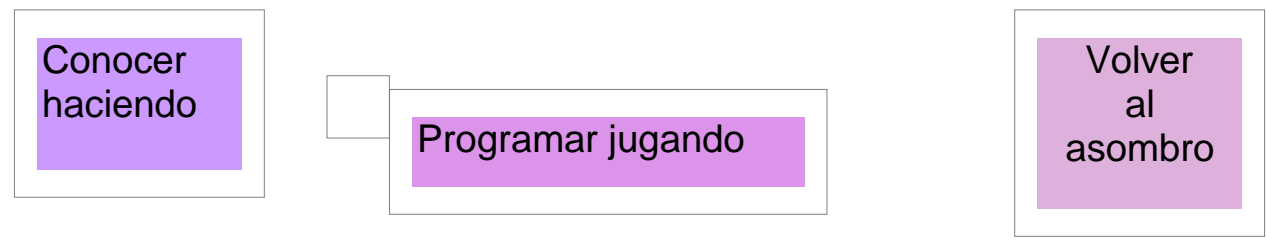
Me siento como pegado, (no mucho) en la puerta de entrada a un aventura interesante.

Sin embargo, algunas de las personas matriculadas pusieron expectativas en la tecnología en sí misma y en aprender a utilizarla, de manera que les ayudara a continuar enseñando a sus estudiantes.

\section{Valoración}

Al finalizar el taller, y por medio de una encuesta en línea que completaron 14 de los 16 de los participantes, se evaluaron diversos aspectos de la realización del taller.

La mayoría (78\%) consideró que se cumplieron los objetivos establecidos en el programa. En la misma forma, la mayoría (93\%) expresó que los contenidos aprendidos 
durante el taller fueron pertinentes para su labor docente, y un $86 \%$ opinó que podría adaptar lo aprendido para mediar el aprendizaje de sus estudiantes.

En cuanto a la convocat9oria y los aspectos de organización de la actividad, la mayor parte (86\%) los consideraron satisfactorios, y un porcentaje similar (85\%) estuvo satisfecho con el trabajo realizado por el equipo facilitador y expresó que recomendaría tomar este taller a otros funcionarios docentes.

El aspecto que fue menos apreciado fue el espacio físico; solamente un 35\% consideró que el espacio físico era apto para la realización de la actividad. La ubicación del mobiliario, las computadoras, y otros equipos no facilitaban el tráfico visual ni el movimiento de los y las participantes, y era casi imposible la reconfiguración para el trabajo en grupos pequeños o plenarias.

La calificación general que hicieron los participantes del taller fue positiva; en la escala de $1-10$, un $43 \%$ lo calificó con un 10 , un $21 \%$ con un 9 , un $21 \%$ con un 8 , y solamente un $14 \%$ con calificación de 7 o menor.

La encuesta incluía una sección abierta sobre observaciones y sugerencias, y en esta se documentaron opiniones que se resumen a continuación.

En su gran mayoría, el entusiasmo y las buenas opiniones expresadas por los docentes universitarios participantes evidencia un nivel de buena acogida y complacencia. Sus apreciaciones en torno a los elementos que rodearon la dinámica, la pertinencia temática desarrollada y los resultados fueron calificados de forma positiva. Por un lado, se tomó la opinión de muchos de ellos y ellas informalmente durante los recesos, así como en la exposición de sus proyectos. La opinión generalizada fue que el taller les trajo una serie de ideas que podrían desarrollar igualmente en clase con sus estudiantes. De esta manera, se alcanzó el objetivo original de modelaje para su posterior implementación por parte de los participantes en sus ambientes específicos de formación. 


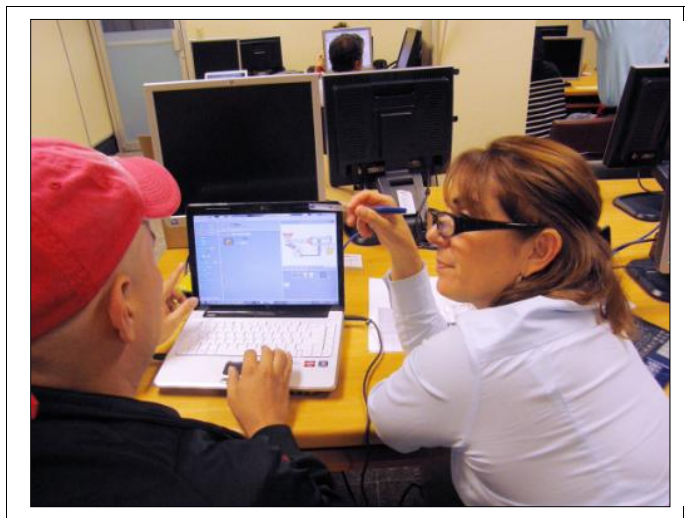

Figura No.7. Proyecto de simulación en desarrollo. grupo de historia digital.

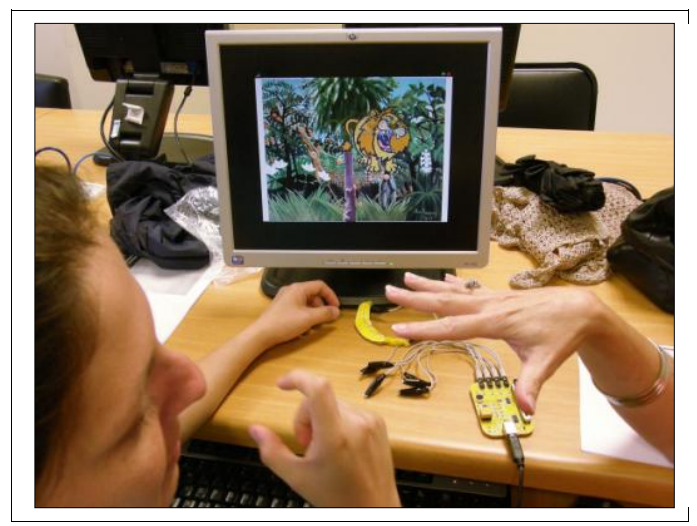

Figura No.8. Discusión realizada en

En otros casos, la inquietud principal fue la de encontrar alguna vía de seguimiento de espacios como el taller. Fue un sentir general la necesidad de contar con espacios de participación y experimentación para los docentes, elemento no tradicional en su rutina dentro de la Universidad de Costa Rica.

Este aspecto toma especial relevancia puesto que permite el planteamiento de nuevos escenarios de desarrollo académico, iniciativas institucionales novedosas que permitirían complementar y continuar la discusión y resultados de iniciativas varias. Estos deberían ser considerados en la lógica de las estructuras de red con las cuales ya se cuenta desde el seno de la Red Institucional de Formación y Evaluación Docente RIFED, entre otras.

De la misma forma, se dio el caso de un participante que expresó insatisfacción en la mayoría de los aspectos evaluados, lo cual justificó diciendo que:

"no hubo material de apoyo en sí de la herramienta; es como si a uno le dijeran vamos a hacer un taller de Access, ahí está la herramienta, ahora úsela y vea cómo se aprende. Así no es".

Debido a que el diseño del taller incluyó apoyo constante de los facilitadores, por medio de demostraciones y trabajo uno a uno, y se complementó con materiales de apoyo disponibles sobre la herramienta Scratch en línea, el comentario se interpretó como una reacción a la costumbre de participar en ambientes de aprendizaje tradicionales, más estructurados, fundamentados en la teoría, y lineales. En este caso, la visión construccionista no fue comprendida ni aceptada por el (la) participante. Esta posición resulta interesante 
puesto que podría retratar la distancia que guardó el taller lejos de la lógica instrumental expresa, en especial por el hecho que la intencionalidad planteada inicialmente buscó desarrollar una clase no para aprender a usar Scratch, sino reflexionar en la concepción de nuevas estrategias docentes y claro está, utilizar elementos para pensar. La herramienta fue escogida como un medio idóneo para que los participantes pudieran repensarse en un contexto.

En general es esperable que un porcentaje de personas reaccionen de manera defensiva contra las innovaciones, ya que prefieren la zona de confort de lo conocido.

Uno de los resultados más positivos del taller fue el tipo de proyecciones que expresaron los y las participantes a partir de la experiencia, las cuales se documentaron por medio de entrevistas. Por ejemplo, se dio un caso en el cual el taller permitió a una persona encontrar en Scratch la herramienta necesaria para estudiar el desarrollo de los procesos de paz en jóvenes de poblaciones vulnerables del país, como parte de una investigación de doctorado. Varios participantes encontraron en Scratch una herramienta que desean utilizar para facilitar el aprendizaje a estudiantes de programación. Aunque el taller no se limitó a presentar Scratch y su funcionamiento, sino que, tal como se explicó anteriormente, fue lo que permitió la inmersión en un ambiente construccionista; de igual forma, es valioso que la herramienta en sí se haya conocido e identificado por su utilidad en diversos contextos.

Las entrevistas complementaron los datos de la encuesta y coincidieron en la opinión favorable de los participantes y el reconocimiento de una nueva forma de aprender, por ejemplo en el siguiente comentario:

"El taller me ha parecido muy interesante, es sentirse libre para crear, y han cumplido la promesa, me ha cambiado la forma de ver lo que es aprender".

En síntesis, es válido decir que el profesorado de la UCR que participó en esta experiencia construccionista valoró positivamente la realización de actividades de este tipo, no solamente por lo expresado anteriormente, sino porque permite la interacción de profesores y profesoras de diversas disciplinas y unidades académicas, y por el nivel de libertad para el aprendizaje y la creación que ofrece el enfoque construccionista. 


\section{Agradecimientos y reconocimientos}

Nuestro agradecimiento a la Dra. Libia Herrero, Vicerrectora de Docencia de la UCR, por su apoyo incondicional en todos los aspectos importantes para la realización del taller. Al Dr. Mitchel Resnick, quien también apoyó al taller, y financió por medio del Lifelong Kindergarden Group, la venida de la Dra. Claudia Urrea al país. A Bárbara Ocampo, Stephanie Fallas, y Armando Vargas, por su importante e imprescindible colaboración en la organización, divulgación, logística y documentación del taller. Las fotografías son de Bárbara Ocampo.

\section{Referencias}

Badilla, Eleonora y Chacón, Alejandra. (2004). Construccionismo: objetos para pensar, entidades públicas y micromundos. Revista Actualidades Investigativas en Educación, 4(1). Recuperado de http://revista.inie.ucr.ac.cr/articulos/12004/archivos/construccionismo.pdf

Bers, Marina. (2001). Identity construction environments: developing personal and moral values through the design of a virtual city. The Journal of the Learning Sciences 10(4), 365-415.

Bruckman, Amy. (1998). Community support for constructionist learning. Computer Supported Cooperative Work, 7, 47-86.

Cabero, Julio. (2006). Bases pedagógicas del e-learning. Revista de Universidad y Sociedad del Conocimiento, 3(1), 1-10.

Flabel, Aaron. (1990). Construccionismo. Fundación Omar Dengo. Traducción libre Eleonora Badilla Saxe. Recuperado de http://llk.media.mit.edu/projects/panama/lecturas/Falbel-Const.pdf

Maloney, John, Resnick, Mitchel, Rusk, Natalie, Silverman, Brian, y Eastmond, Evelyn. (2010). The Scratch Programming Language and Environment. ACM Transactions on Computing Education (TOCE), 10(4). Recuperado de http://web.media.mit.edu/ imaloney/papers/ScratchLangAndEnvironment.pdf

Seneviratne, Oshani, Monroy-Hernández, Andrés. (2010). Remix Culture on the Web: A Survey of Content Reuse on Different User-Generated Content Websites. In Web Science. http://journal.webscience.org/392/

Papert, Seymour. (1990). A Critique of Technocentrism in Thinking About the School of the Future, en http://www.papert.org/articles/ACritiqueofTechnocentrism.html

Papert, Seymour. (1987). Desafío de la mente. Buenos Aires, Argentina: Ediciones Galápagos. 
Papert, Seymour. (2000). What's the Big Idea? Steps toward a pedagogy of idea power. IBM Systems Journal, 39(3y4), 720-729.

Papert, Seymour, y Resnick, Mitchel. (1995). Technological fluency and the representation of knowledge. Proposal to the National Science Foundation. Cambridge, MA.

Resnick, Mitchel. (1994). Turtles, termites, and traffic jams: explorations in massively parallel microworlds. Cambridge, Mass.: MIT Press.

Resnick, Mitchel, et al. (2009). Scratch: programming for all. Commun ACM, 52(11), 60-67.

Resta, Paul. (2004). Las tecnologías de la información y la comunicación en la formación docente: Guía de planificación. Montevideo, Uruguay: Ediciones Trilce.

Stevens, Vanessa, Klopfer, Eric y Resnick, Mitchel. (2001). Adventures in modeling: exploring complex, dynamic systems with StarLogo. New York, USA: Teachers College Press. 


\section{Anexo No.1}

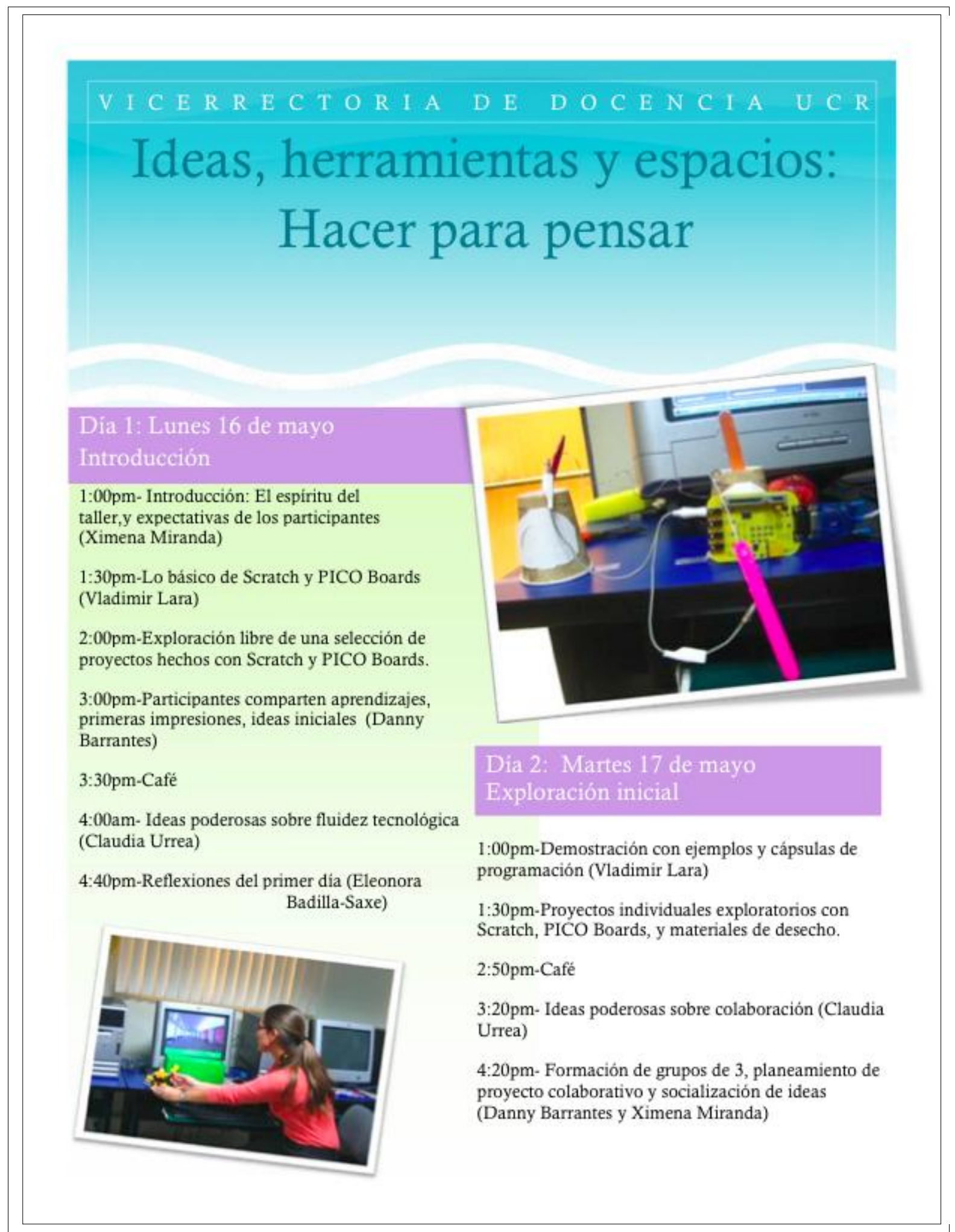

Volumen 12, Número 1, Año 2012, ISSN 1409-4703 
TALLER IDEAS, HERRAMIENTAS Y ESPACIOS: HACER PARA PENSAR

\section{Día 3: Jueves 19 de mayo. Creación de un proyecto colaborativo}

1:00pm- Demostración con ejemplos y cápsulas de programación (Vladimir Lara)

1:30pm-Inicio de proyectos colaborativos libres.

3:00pm-Café

3:20pm-Grupos muestran avance de sus proyectos y comparten sus aprendizajes, emociones, preguntas, dificultades. Preguntas: ¿Cómo fue el proceso de colaboración? ¿Podrían compartir algunos ejemplos específicos de cada grupo? (Ximena Miranda y Danny Barrantes)

4:40am- Reflexiones del tercer día: la docencia construccionista (Eleonora Badilla-Saxe).

\section{Día 4: Viernes 20 de mayo. Participación en evento mundial "Scratch Day"}

1:00pm-Scratch Day y la comunidad internacional de Scratch (Ximena Miranda).

1:30pm- Tiempo para terminar los proyectos colaborativos y colocarlos en la página web de Scratch.

2:30pm- Grupos muestran avance de sus proyectos y comparten sus aprendizajes, emociones, preguntas, dificultades (Danny Barrantes)

3:30pm- Café

3:50pm- Reconstrucción del proceso vivido en el taller ,reflexiones finales, y proyecciones (Eleonora BadillaSaxe) 